\title{
Model Based Early Kick/Loss Detection and Attenuation with Topside Sensing in Managed Pressure Drilling
}

\author{
Asanthi Jinasena ${ }^{1}$ Haavard Holta ${ }^{2} \quad$ Morten Hansen Jondahl $^{3} \quad$ Prasanna Welahettige $^{4}$ Ole Morten \\ $\mathrm{Aamo}^{2}$ Roshan Sharma ${ }^{3}$ Håkon Viumdal ${ }^{3}$ Bernt Lie ${ }^{3}$ \\ ${ }^{1}$ Department of Energy and Process Engineering, Norwegian University of Science and Technology, Norway, \\ asanthi.jinasena@ntnu. no \\ ${ }^{2}$ Department of Engineering Cybernetics, Norwegian University of Science and Technology, Norway, \\ \{haavard.holta, aamo\} entnu. no \\ ${ }^{3}$ Department of Electrical Engineering, Information Technology and Cybernetics, University of South-Eastern \\ Norway, Norway, morthans1@gmail.com, \{roshan.sharma, Hakon.Viumdal, Bernt. Lie\}@usn.no \\ ${ }^{4}$ Department of Process, Energy and Environmental Technology, University of South-Eastern Norway, Norway, \\ prasanna. welahetti@gmail. com
}

\begin{abstract}
Early kick/loss detection is a crucial part of safe well control, and it plays a major role in the reduction of risk and non-productive time in drilling. In conventional drilling, topside sensing is used for early kick/loss detection. Recently, Venturi flowmeter based online return flow estimation has been introduced for this purpose by the authors. In managed pressure drilling, both topside sensing and bottomside sensing can be used for kick/loss detection. Therefore, a topside return flow estimator with a bottomside well pressure and flow estimator is combined to provide a complete kick/loss detection and estimation scheme for managed pressure drilling systems. This allows improved kick/loss detection. In addition, a closedloop kick/loss attenuation controller is used to illustrate the estimation scheme.

Keywords: kick loss detection, managed pressure drilling $(M P D)$, return flow, adaptive control, UKF
\end{abstract}

\section{Introduction}

\subsection{Background}

Oil and gas drilling is done by penetrating a rotating drill bit into the rock formation, creating a wellbore. The formation is a high pressure and temperature environment. Therefore, a drilling fluid known as mud is continuously circulated through the wellbore. This circulation process is usually divided into two parts: bottomside (wellbore) and topside (section on the surface).

The bottomhole pressure (BHP) is maintained within a window for safe operation. If BHP is lower than the formation fluid pressure, formation fluid may enter the wellbore. This phenomenon is commonly referred to as a 'kick' which could result in a catastrophic blowout if not controlled properly. If BHP is higher than the fracture pressure of the formation, the drilling mud may seep into the formation, which is known as a 'loss'.
Early kick/loss detection is a crucial part of safe well control, and it plays a major role in the reduction of risk and non-productive time in drilling. In conventional drilling, this is primarily achieved by using the topside data, mainly the return mud flow measurement, and the volume gain in the mud pit. For the return flow, costeffective, accurate and online sensors are needed in this regard. For applications with narrow pressure margins, a control choke and a back-pressure pump are used to control the BHP fast and accurately. This is known as managed pressure drilling (MPD).

The topside sensing includes among other measurements: return flow measurements, mud pit gains, and other respective rheological properties of the drilling mud that is essential for normal drilling operations. However, the rheological measurements are often offline, manual measurements with low frequencies and not eligible for automation purposes.

\subsection{Previous Work on Topside Sensing}

A Venturi flowmeter based online return flow estimation has been studied recently. The fluid level changes in the open channel is measured non-intrusively and used to model the fluid flow rate. Both mechanistic and datadriven models are used for this purpose (Berg et al., 2015; Chhantyal et al., 2018; Jinasena et al., 2018; Welahettige, 2019; Jinasena, 2019; Jondahl, 2020; Berg, 2020). Further, these fluid level characterizations are used as inputs to different data-driven models for the estimation of the drilling fluid rheological properties (Chhantyal et al., 2016). Moreover, some of the fluid rheological properties which are essential for the drilling operation (mainly viscosity and density) are estimated using ultrasonic waves in a stationary medium (Jondahl and Viumdal, 2018, 2019; Jondahl, 2020). The estimations were done by using different data-driven models, and the estimation errors were within the NORSOK standards accuracy of $2 \%$ (Jondahl, 2020). 
Further, the viscosity and the flow behaviour of the drilling mud has been studied extensively using 3D computational fluid dynamics (CFD) simulations (Welahettige, 2019). This has been beneficial to the different models that can be used for return flow rate estimation. High-resolution numerical schemes such as the fluxlimited-centered scheme has been applied to solve a nonNewtonian model for open channel. This scheme is wellbalanced, positivity-preserving and has a high accuracy, and a good resolution for discontinuities. The developed model was tested with hydraulic jumps propagation in open Venturi channels. Moreover, the effect of drill cuttings on the return flow measurements have also been studied (Welahettige et al., 2019) and a multi-fluid volume of fluid model has been used for the CFD analyses.

These studies as a whole, improve the topside models and the estimations. Further, these studies complement the topside sensing and the early kick detection in general.

In this paper, we combine a topside return flow estimator with a bottomside well pressure and flow estimator to provide a complete kick/loss detection and estimation scheme for MPD systems. In addition, to illustrate the estimation scheme, a closed loop kick/loss attenuation controller is used.

The paper is organized as follows. The complete system, including the mathematical models, estimation and control methods, are presented in Section 2. This is followed by the simulation set-up, detailed results and discussion in Section 3. Finally, the conclusions drawn from the results and discussion are summarized in Section 4. Further, a more detailed mathematical overview of the estimator schemes can be found in the Appendix A.

\section{System Description}

The block diagram of the considered complete flow loop of the MPD system is shown in Figure 1. The main process steps of the entire flow loop which are modeled are shown here with the notations.

\subsection{Mathematical Models}

Both the flow dynamics in the annulus and the top-side Venturi channel are modelled as distributed systems of hyperbolic PDEs, while the drill string is considered as a known input-output system. In the annulus, the pressure $p(z, t)$ and flow rate $q(z, t)$ are modelled by the well known water hammer equations for compressible, single-phase flows with low Mach numbers (Ghidaoui et al., 2005), while the wetted cross-sectional area $A_{c}(x, t)$ and flow rate $q_{l}(x, t)$ in the Venturi channel are modelled by the SaintVenant, shallow water equations (Chow, 1959; Chaudhry, 2008).

The water hammer equations modelling the well flow have the form,

$$
\frac{\partial p(z, t)}{\partial t}=\frac{\beta}{A_{\mathrm{a}}} \frac{\partial q(z, t)}{\partial z}
$$

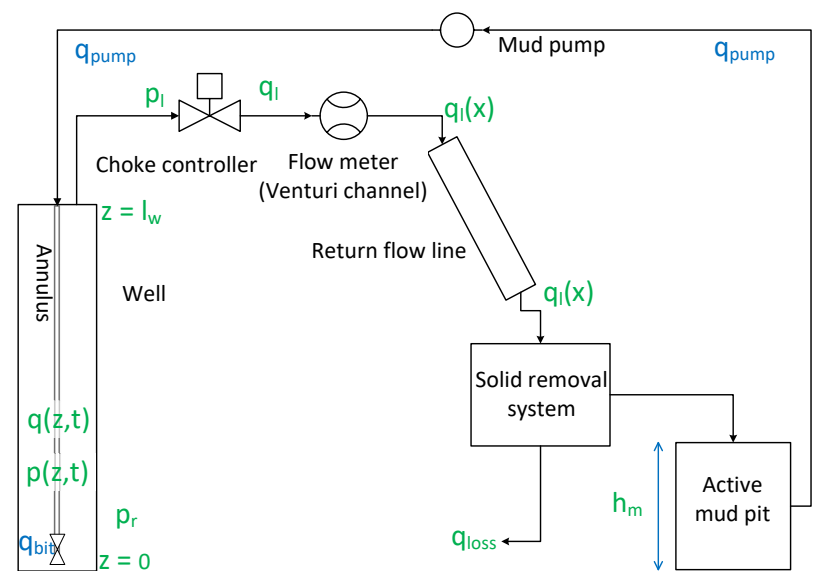

Figure 1. The block diagram of the flow loop of a MPD system.

$$
\frac{\partial q(z, t)}{\partial t}=-\frac{A_{\mathrm{a}}}{\rho} \frac{\partial p(z, t)}{\partial z}-\frac{F_{\mathrm{a}}}{\rho} q(z, t)-A_{\mathrm{a}} g .
$$

Here, $z \in\left[0, l_{\mathrm{w}}\right]$ and $t \geq 0$ are the independent variables of space and time respectively, where $z=l_{\mathrm{w}}$ is at the top of the well and $l_{\mathrm{w}}$ is the well depth. $\rho$ is the density of the drilling mud, $\beta$ is the bulk modulus of the mud, $F_{\mathrm{a}}$ is the friction factor in the annulus, $A_{\mathrm{a}}$ is the cross sectional area of the annulus and $g$ is the acceleration of gravity.

The two boundary conditions are the topside pressure $p\left(l_{\mathrm{w}}, t\right)$, and the net inflow $q(0, t)$ at the bottom of the well. The topside pressure $p_{l}(t)$ is related to the topside flow rate $q\left(l_{w}, t\right)$ through the choke equation,

$$
q\left(l_{w}, t\right)=k_{\text {choke }}(t) \operatorname{sign}\left(p_{l}(t)-p_{0}\right) \sqrt{\left|p_{l}(t)-p_{0}\right|},
$$

where $p_{0}$ is the atmospheric pressure and $k_{\text {choke }}$ is the choke coefficient which can be used as an actuation. The net inflow is modelled as a simple linear flow-pressure relationship as follows (Dake, 1998),

$$
\begin{aligned}
q(0, t) & =J\left(p_{\mathrm{r}}-p(0, t)\right)+q_{\mathrm{bit}}, \\
p\left(l_{\mathrm{w}}, t\right) & =p_{\mathrm{l}}(t),
\end{aligned}
$$

where $p_{\mathrm{r}}$ is the pressure in the surrounding formation, $q_{\mathrm{bit}}$ is a known volumetric flow through the drill bit, $J$ is the so-called productivity-index. Both the productivity index $J>0$, and the formation pressure $p_{r}>0$ are assumed unknown and must be estimated. The topside flow rate $q_{l}(t)$ is modeled using the topside model which is described next.

The topside flow rate $q_{l}(x, t)$ is measured by a Venturi channel flowmeter, which uses the fluid levels $h(x, t)$ to calculate the flow rate based on the 1-D shallow water equations (Chow, 1959; Chaudhry, 2008).

$$
\frac{\partial A_{\mathrm{c}}(x, h, t)}{\partial t}=-\frac{\partial q_{l}(x, t)}{\partial x}
$$




$$
\begin{aligned}
\frac{\partial q_{l}(x, t)}{\partial t}= & -\frac{\partial}{\partial x}\left(\alpha \frac{q_{l}^{2}(x, t)}{A_{\mathrm{c}}(x, h, t)}+g I_{1} \cos \phi\right)+g I_{2} \\
& +g A_{\mathrm{c}}(x, h, t) \sin \phi-T_{\mathrm{f}}
\end{aligned}
$$

Here, $A_{\mathrm{c}}(x, h, t)$ is the wetted cross sectional area in the channel normal to the flow, $h(x, t)$ is the depth of flow, and $q_{l}(x, t)$ is the volumetric flow rate in the channel. $x \in\left[0, l_{\mathrm{c}}\right]$ is the position along the channel, and $l_{\mathrm{c}}$ is the length of the channel. $I_{1}$, the first moment of area represents the hydrostatic pressure term and $I_{2}$ represents the pressure forces in the fluid volume, which occur from the longitudinal width and slope variations in the channel. $\phi$ is the channel bottom slope angle, $\alpha$ is known as the momentum correction coefficient or the Boussinesq coefficient and corresponds to the deviations of the local velocity over the mean velocity of the flow, and $T_{f}$ is the non-Newtonian friction term.

Equations 6 and 7 are used for the Venturi channel with a trapezoidal cross section and a zero bottom slope angle. The boundary condition for the Venturi channel is the topside flow rate $q\left(l_{w}, t\right)$ of the choke equation (equation 3 ). The fluid levels produced by the model will be then taken as measurements for the estimation.

The same set of equations ( 6 and 7 ) is used to model the return flowline with a circular cross section and a $7^{\circ}$ bottom slope angle. The flowline is connected to the Venturi by taking the Venturi outlet flow rate $q_{l_{3}}(t)$ as the boundary condition to the flowline model. More details on the Venturi channel model and the return flowline model can be found at (Jinasena et al., 2019; Pirir et al., 2018), respectively.

The active mud pit level $h_{\mathrm{m}}$ is modeled by the simplified mass balance equation as follows (Pirir et al., 2018),

$$
\frac{\mathrm{d} h_{\mathrm{m}}}{\mathrm{d} t}=\frac{1}{A_{\mathrm{m}}}\left(q_{l}-q_{\text {loss }}-q_{\text {pump }}\right),
$$

where $A_{\mathrm{m}}$ is the cross sectional area of the active mud pit, $q_{\text {loss }}$ is the fluid losses at solid removal equipment and $q_{\text {pump }}$ is the mud flow in to the well which is measured by the mud pump. Here $q_{l}$ is the outlet flow rate of the flowline.

The density of the drilling mud is assumed to be constant throughout the flow loop, and only a single phase (liquid) flow is considered with no gas or cuttings flow.

The return flow rate from the Venturi channel, and the mud pit level are estimated. Further, the topside model allows to estimate the non-Newtonian friction coefficient of the mud and the fluid losses from the solid removal equipment as well (Jinasena et al., 2019; Jinasena and Sharma, 2020). However, the friction coefficient and fluid losses are not estimated in this study as the focus is on the well dynamics.

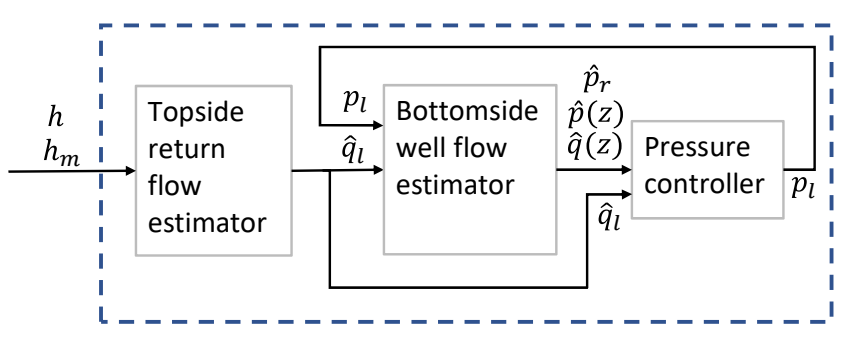

Figure 2. Estimators and controller configuration.

\subsection{Estimation and Control Methods}

The main objective of this study is to use this combined model to estimate the reservoir pressure and the BHP, which can be an input to the kick/loss attenuation system.

The system configuration is shown in Figure 2. The available measurements for the system are the fluid levels of the Venturi channel and the mud pit level. The topside estimator uses both of these measurements to estimate the return flow rate. The return flow estimates are used as an input to the bottomside estimator, where the annulus pressure and flow, and the reservoir pressure and productivityindex are estimated. All estimated states and parameters are then used to guide a closed-loop kick/loss attenuation system by adjusting the top-side MPD choke opening.

In this study, for the topside estimator, we use the estimator presented in (Jinasena et al., 2019) which is based on an unscented Kalman filter (UKF). For the bottomside estimator we use the adaptive observer design presented in (Holta et al., 2017).

\subsubsection{UKF}

The return flow rate, the non-Newtonian friction coefficient and the rate of fluid loss at the solids removal system can be estimated by the UKF (Jinasena and Sharma, 2020). One of the fluid levels of the Venturi channel can be used as an input, if preferred. However, in this study, two fluid levels are used as measurements and no input is used for the estimator. Further, only the return flow rate is estimated for simplicity. More details on the UKF can be found in (Jinasena et al., 2019; Jinasena and Sharma, 2020) and a brief explanation is given in Appendix A.2.

\subsubsection{Adaptive Observer}

The distributed pressure $p(z, t)$ and flow $q(z, t)$ in the annulus, are estimated using a distributed-state observer which is derived using the so-called infinite-dimensional backstepping design. The unknown reservoir pressure $p_{\mathrm{r}}$ and the productivity index $J$ are estimated by an adaptive law based on a linear parametric model. Both the topside choke pressure and the estimated return flow are considered as external inputs to the observer. All necessary design elements needed to implement the adaptive observer are provided in Appendix A.1. More details on the observer design, including stability proofs can be found in (Holta et al., 2017). 


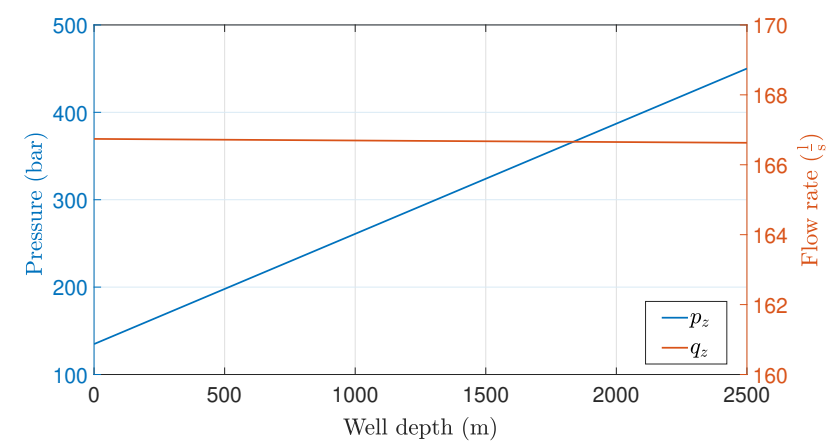

Figure 3. The pressure $p_{z}$ and flow rate $q_{z}$ along the well depth.
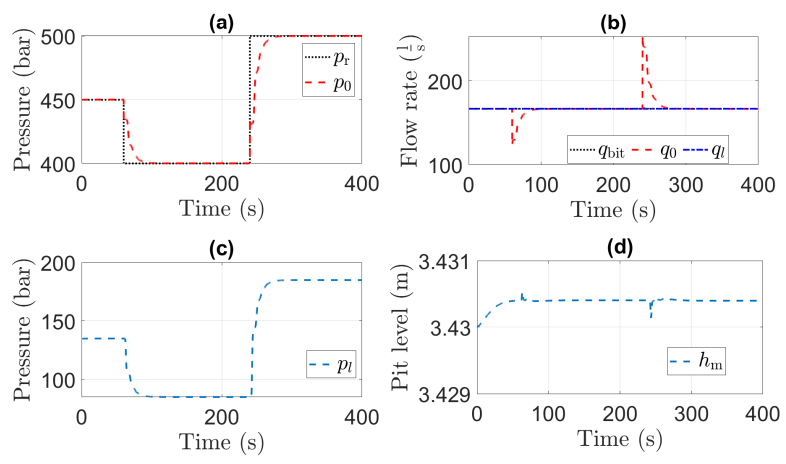

Figure 4. The model results, (a) reservoir pressure $p_{\mathrm{r}}$ and well pressure $p_{0}$, (b) flow through bit $q_{\text {bit }}$, bottom hole flow rate $q_{0}$ and return flow rate $q_{l}$, (c) control signal $p_{l}$, and (d) mud pit level $h_{\mathrm{m}}$.

\subsubsection{The Choke Controller}

Based on the return flow estimates, the well flow and pressure estimates, and the estimated reservoir properties, a desired topside choke pressure is computed by the kick/loss attenuation system. The kick/loss attenuation system is derived using the infinite-dimensional backstepping approach. In (Holta et al., 2017), stability is proved for the closed loop system consisting of the bottomside estima-tor and the pressure controller. From the choke equation (Equation 3), the variable choke opening $k_{\text {choke }}(t)$ can be adjusted to match a given return flow estimate and desired topside pressure.

\section{Results and Discussion}

The bottomside model and estimator are implemented in MATLAB using a $1^{\text {st }}$ order upwind scheme for the spatial discretization and Euler scheme for the temporal discretization. The topside model and estimators are implemented in MATLAB using orthogonal collocation for the spatial discretization and Runge-Kutta $4^{\text {th }}$ order scheme for the temporal discretization.

The simulation results of the well model, mainly the pressure $p_{z}$ and flow rate $q_{z}$ for constant input values can be seen from Figure 3. The linear relationship of pressure with the well depth can be clearly seen.

The simulation results of the combined model with a

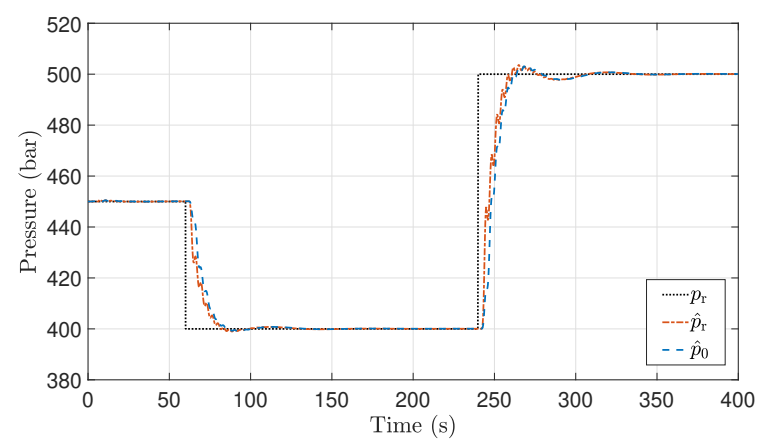

Figure 5. The estimated reservoir pressure $\left(\hat{p}_{\mathrm{r}}\right)$ and BHP $\left(\hat{p}_{0}\right)$ with the actual reservoir pressure $\left(p_{\mathrm{r}}\right)$.

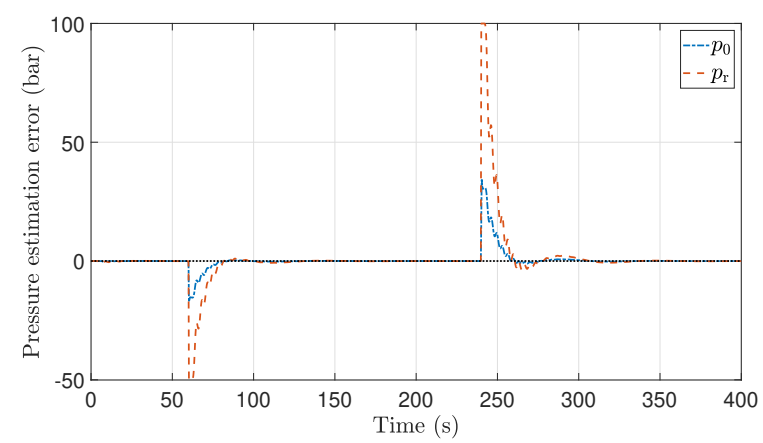

Figure 6. The estimation error of $\operatorname{BHP}\left(p_{0}-\hat{p}_{0}\right)$ and reservoir pressure $\left(p_{\mathrm{r}}-\hat{p}_{\mathrm{r}}\right)$.

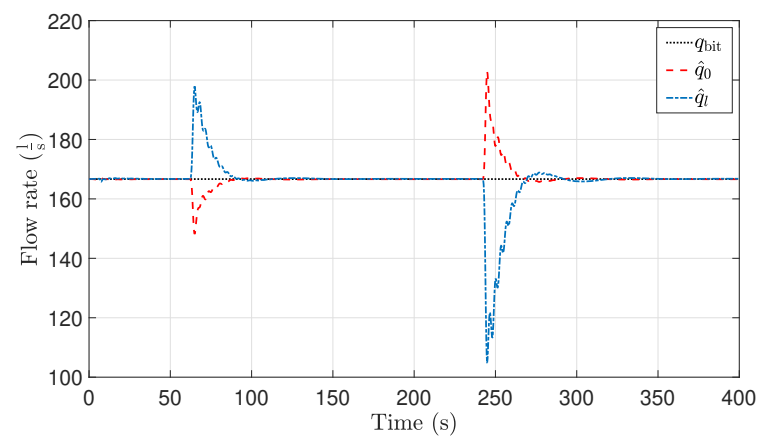

Figure 7. The estimated volumetric flow rates $\hat{q}_{0}$ and $\hat{q}_{l}$ against the flow through drill bit $q_{\text {bit }}$.

known reservoir pressure, and known flow through bit are shown in Figure 4. The objective of keeping the BHP close to the reservoir pressure is achieved with a small time lag of about $40 \mathrm{~s}$ as shown in (a) in Figure 4. Further, the control signal and the mud pit volume are shown.

The estimated BHP and the reservoir pressure using the bottomside adaptive observer are shown in Figure 5 together with the actual reservoir pressure.

The estimation error between the estimated BHP and the actual BHP is shown in Figure 6 as well as the estimation error between the estimated and actual reservoir pressure. The estimation error of the reservoir pressure is quite high due to the time lag. However, the BHP estimation error is comparatively low. 


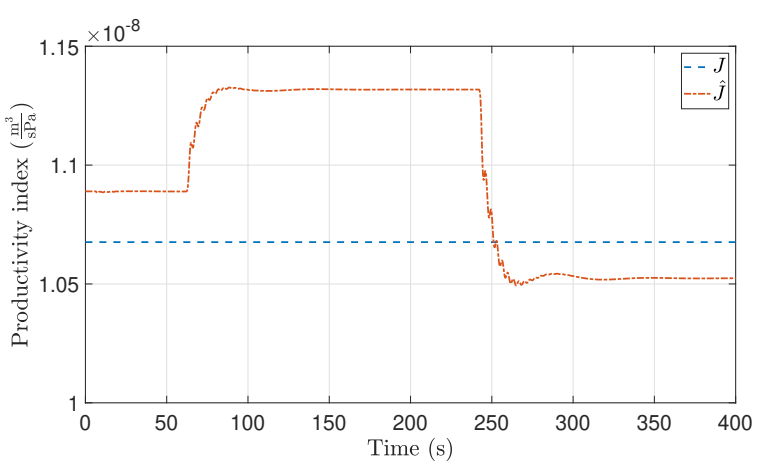

Figure 8. The estimated productivity index $\hat{J}$ and the constant productivity index $J$ that is used in the model.

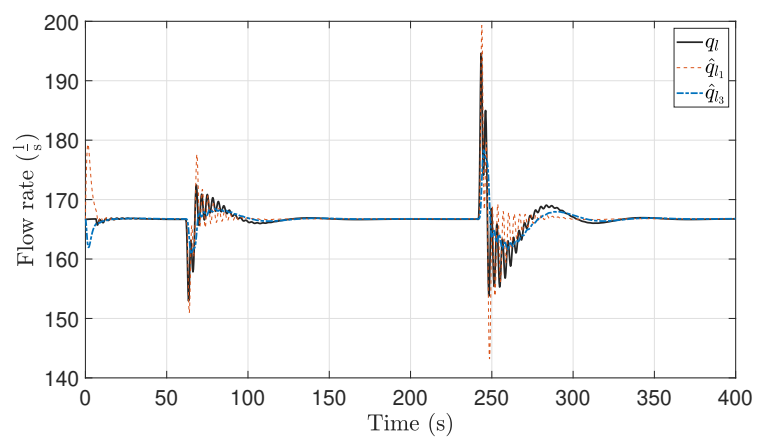

Figure 9. The estimated volumetric flow rates along the Venturi channel $\left(\hat{q}_{l_{1}}\right.$ and $\left.\hat{q}_{l_{3}}\right)$ with the actual flow rate $q_{l}$.

Similarly, the observer results of the estimated volumetric flow rates at bottom and top of the well are shown in Figure 7 together with the volumetric flow rate through the drill bit. The change of the estimated parameter, productivity index, with the change of reservoir pressure can be seen in Figure 8 against the constant value used in the model.

The topside estimator results of the volumetric flow rates along the Venturi channel are shown in Figure 9 with the model result from the bottomside model. The numerical oscillations on the actual flow rate are filtered out by the UKF along the channel length as seen on the flow rate estimation of the third position of the channel. This flow rate is then used in the active mud pit model to estimate the mud pit level. The estimated mud pit level is shown in Figure 10 with the actual mud pit level. Since the flow rate fluctuation during disturbances are quite low, the mud pit level fluctuation is also comparatively low.

The estimation errors of different flow rate estimates are shown in Figure 11. The estimation error of the flow rate at the bottom of the well and the estimation error of flow rate at the top of the well are quite high similar to the pressure estimation error of the observer. However, the estimation error of the Venturi flow rate is comparatively low. Although the high estimation errors exist when considered separately, the estimated flow rate at the top of the well (return flow rate) for the complete system is taken to be the estimated Venturi channel flow rate.

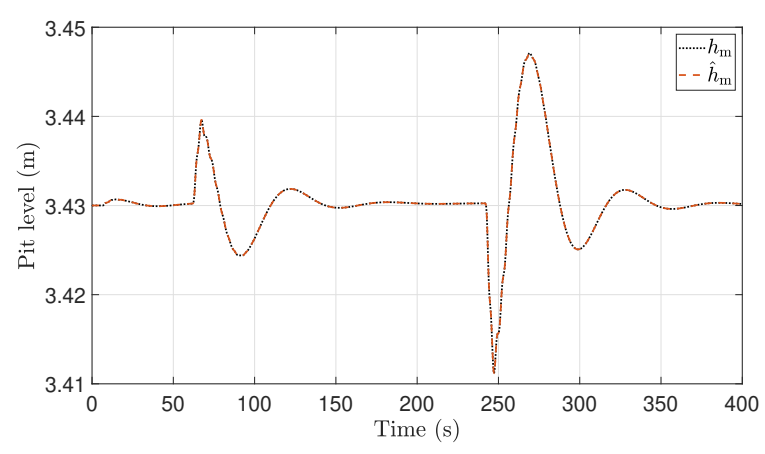

Figure 10. The estimated active mud pit level $\hat{h}_{\mathrm{m}}$ with the actual mud pit level $h_{\mathrm{m}}$.

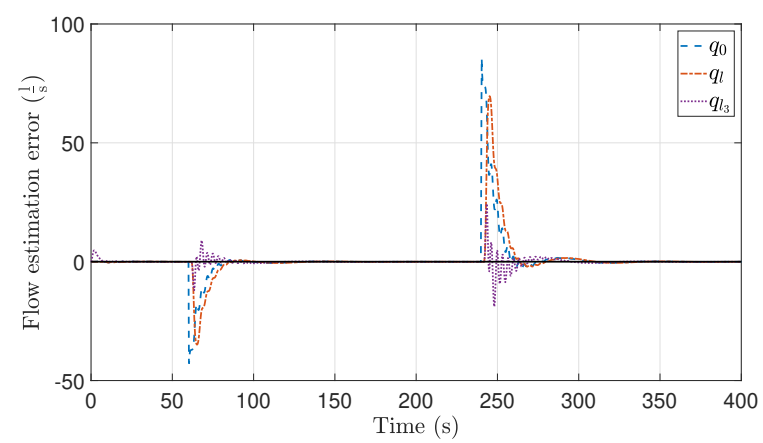

Figure 11. The estimation error of flow rate at bottom of the well $\left(q_{0}-\hat{q}_{0}\right)$, flow rate at top of the well $\left(q_{l}-\hat{q}_{l}\right)$ and flow rate at the Venturi channel $\left(q_{l}-\hat{q}_{l_{3}}\right)$, respectively.

\section{Conclusions}

The complete flow loop of a managed pressure drilling system is tested for a novel, mathematical model-based kick/loss detection scheme. A topside return flow estimator is combined with a bottomside well pressure and flow estimator to provide this complete kick/loss detection scheme. The topside flow rate estimator consists of a Venturi flowmeter, return flowline and the active mud pit. This return flow estimate is then used as input to the bottomside wellbore estimator. The distributed pressure and flow rate in the annulus, and the unknown reservoir pressure and the productivity index are estimated by an adaptive observer. The topside and bottomside estimators are then used in closed loop with a topside choke controller. The bottomhole pressure is controlled to be equal to the estimated reservoir pressure and the kick/loss is attenuated in a simulation environment. The estimation results are accurate and show improved kick/loss detection and attenuation capability.

\section{Acknowledgement}

The economic support from the Research Council of Norway and Equinor ASA through project no. 255348/E30 'Sensors and models for improved kick/loss detection in drilling (Semi-kidd)' is gratefully acknowledged. 


\section{A Appendix}

The details of the estimators are briefly stated in this section.

\section{A.1 Adaptive Observer}

The system described in equations 1 - 5 can be written in an equivalent characteristic form using its Riemann coordinates $(u, v)$, which are obtained through a linear transformation of variables $(p, q) \rightarrow(u, v)$ (see for example (Aamo, 2013)):

$$
\begin{aligned}
\frac{\partial u\left(z_{\mathrm{n}}, t\right)}{\partial t}+\lambda \frac{\partial u\left(z_{\mathrm{n}}, t\right)}{\partial z_{\mathrm{n}}} & =c_{1}\left(z_{\mathrm{n}}\right) v\left(z_{\mathrm{n}}, t\right) \\
\frac{\partial v\left(z_{\mathrm{n}}, t\right)}{\partial t}-\mu \frac{\partial v\left(z_{\mathrm{n}}, t\right)}{\partial z_{\mathrm{n}}} & =c_{2}\left(z_{\mathrm{n}}\right) u\left(z_{\mathrm{n}}, t\right) \\
u(0, t) & =\theta_{1} v(0, t)+\theta_{2} \\
v(1, t) & =U(t) \\
y(t) & =u(1, t)
\end{aligned}
$$

Here, $z_{\mathrm{n}} \in[0,1]$ and $t \geq 0$ are the independent variables, while $u\left(z_{\mathrm{n}}, t\right)$ and $v\left(z_{\mathrm{n}}, t\right)$ are the transformed states of the system. Further, $\lambda, \mu>0$, and $c_{1}\left(z_{\mathrm{n}}\right), c_{2}\left(z_{\mathrm{n}}\right) \in C([0,1])$ are known, while $\theta_{1}, \theta_{2}$ are unknown parameters given uniquely by the unknown reservoir parameters $J, p_{\mathrm{r}} . U(t)$ and $y(t)$ are uniquely given by the topside pressure $p_{l}(t)$ and topside flow $q_{l}(t)$.

The following observer is designed for the system.

$$
\begin{aligned}
\frac{\partial \hat{u}}{\partial t}+\lambda \frac{\partial \hat{u}}{\partial z_{\mathrm{n}}} & =c_{1}\left(z_{\mathrm{n}}\right) \hat{v}+P_{1}\left(z_{\mathrm{n}}, t\right)[y(t)-\hat{u}(1, t)] \\
\frac{\partial \hat{v}}{\partial t}-\mu \frac{\partial \hat{v}}{\partial z_{\mathrm{n}}} & =c_{2}\left(z_{\mathrm{n}}\right) \hat{u}+P_{2}\left(z_{\mathrm{n}}, t\right)[y(t)-\hat{u}(1, t)] \\
\hat{u}(0, t) & =\hat{\theta}_{1} \hat{v}(0, t)+\hat{\theta}_{2} \\
\hat{v}(1, t) & =U(t)
\end{aligned}
$$

Here, $\hat{u}, \hat{v}$ are the system state estimates, $\hat{\theta}_{1}, \hat{\theta}_{2}$ are the parameter estimates and $P_{1}, P_{2}$ are the output injection gains satisfying

$$
\begin{aligned}
& P_{1}(x, t)=\lambda P^{u}(x, 1, t) \\
& P_{2}(x, t)=\lambda P^{v}(x, 1, t)
\end{aligned}
$$

where the kernels $P^{u}, P^{v}$ are the unique solution to the system

$$
\begin{aligned}
P_{t}^{u}(x, \xi, t)+\lambda P_{x}^{u}(x, \xi)+\lambda P_{\xi}^{u}(x, \xi) & =c_{1}(x) P^{v}(x, \xi) \\
P_{t}^{v}(x, \xi, t)-\mu P_{x}^{v}(x, \xi)+\lambda P_{\xi}^{v}(x, \xi) & =c_{2}(x) P^{u}(x, \xi) \\
P^{v}(x, x) & =\frac{c_{2}(x)}{\lambda+\mu} \\
P^{u}(0, \xi) & =\hat{\theta}_{1} P^{v}(0, \xi) .
\end{aligned}
$$

The equations are solved by a finite-difference method over a selected triangular discretized mesh of space.

The parameter estimates $\hat{\theta}_{1}, \hat{\theta}_{2}$ are generated by the adaptive laws

$$
\begin{gathered}
\dot{\hat{\theta}}_{1}(t)= \begin{cases}\Gamma \frac{\vartheta(t)-\bar{v}(t) \hat{\theta}_{1}-\hat{\theta}_{2}}{2+\bar{v}(t)} \bar{v}(t) & \text { for } t>t_{F} \\
0 & \text { otherwise }\end{cases} \\
\dot{\hat{\theta}}_{2}(t)= \begin{cases}\Gamma \frac{\vartheta(t)-\bar{v}(t) \hat{\theta}_{1}-\hat{\theta}_{2}}{2+\bar{v}(t)} & \text { for } t>t_{F} \\
0 & \text { otherwise }\end{cases}
\end{gathered}
$$

where

$$
\begin{aligned}
\vartheta(t)= & y(t)-\hat{u}(1, t)+\hat{\theta}_{1}\left(t-d_{\alpha}\right) \bar{v}(t)+\hat{\theta}_{2}\left(t-d_{\alpha}\right) \\
\bar{v}(t)= & \hat{v}\left(0, t-d_{\alpha}\right) \\
& +\int_{0}^{1} P^{v}\left(0, \xi,-t-d_{\alpha}\right) y\left(t-\frac{\xi}{\lambda}\right) d \xi \\
& -\int_{0}^{1} P^{v}\left(0, \xi,-t-d_{\alpha}\right) \hat{u}\left(1, t-\frac{\xi}{\lambda}\right) d \xi
\end{aligned}
$$

\section{A.2 UKF}

The shallow water equations (equations 6 and 7) can be simplified into ordinary differential equations using the orthogonal collocation (for example using 3 collocation points) as follows.

$$
\frac{\mathrm{d} A_{\mathrm{c}_{i}}}{\mathrm{~d} t}=-\sum_{i=1}^{3} M_{i j}^{T} q_{l_{i}}
$$

$$
\begin{aligned}
\frac{\mathrm{d} q_{l_{i}}}{\mathrm{~d} t}= & -\sum_{i=1}^{3} M_{i j}^{T} \frac{q_{l_{i}}^{2}}{A_{\mathrm{c}_{i}}}-g \sum_{i=1}^{3} M_{i j}^{T} I_{1_{i}} \cos \phi \\
& +g A_{\mathrm{c}_{i}} \sin \phi-T_{\mathrm{f}_{i}} \\
M & =\frac{1}{l_{\mathrm{c}}}\left[\begin{array}{ccc}
-3 & 4 & -1 \\
-1 & 0 & 1 \\
1 & -4 & 3
\end{array}\right],
\end{aligned}
$$

Here $i, j \in[1,2,3]$. The detailed derivation of the equations can be found in (Jinasena et al., 2017). This sim-plified non-linear system for the UKF can be written in discrete time form as follows,

$$
\begin{aligned}
X_{k+1} & =f_{\mathrm{n}}\left(X_{k}, t_{k}\right)+w_{k}\left(t_{k}\right), \\
y_{k} & =C X_{k}+v_{k}\left(t_{k}\right),
\end{aligned}
$$

The function $f_{\mathrm{n}}$ represents the nonlinear model given by Equations (28)-(30) and (8) for $\dot{h}_{i}, \dot{q}_{l_{i}}$, and $\dot{h}_{\mathrm{m}}$, respectively. $k$ is the discrete time index. The measurement noise in the measured output at $t_{k}$ is denoted by $v_{k} \in \mathbb{R}^{n_{\mathrm{m}}}$, where $v_{k} \sim \mathscr{N}(0, R)$. Similarly, $w_{k} \in \mathbb{R}^{n_{\mathrm{s}}}$, where $w_{k} \sim \mathscr{N}(0, Q)$ accounts for process noise. Here, $n_{\mathrm{m}}$ and $n_{\mathrm{s}}$ are the number of measurements and number of states, while $R$ and $Q$ are the covariance matrices for measurement noise and process noise, respectively.

The states and measurements can be chosen from the available fluid levels as preferred, based on the 
necessity and observability. For example; $X=$ $\left[\begin{array}{lllllll}h_{1} & h_{2} & h_{3} & q_{l_{1}} & q_{l_{2}} & q_{l_{3}} & h_{\mathrm{m}}\end{array}\right]^{T}$ and $y=\left[\begin{array}{lll}h_{2} & h_{3} & h_{\mathrm{m}}\end{array}\right]^{T}$ is chosen for faster convergence of the observer, thus $C$ becomes as follows,

$$
C=\left[\begin{array}{lllllll}
0 & 1 & 0 & 0 & 0 & 0 & 0 \\
0 & 0 & 1 & 0 & 0 & 0 & 0 \\
0 & 0 & 0 & 0 & 0 & 0 & 1
\end{array}\right]
$$

The estimated return flow rate $\hat{q}_{l_{1}}$ or $\hat{q}_{l_{3}}$ is then used in the $p_{0}$ bottomside estimator.

\section{Nomenclature}

$\alpha \quad$ Momentum correction coefficient

$\beta \quad$ Bulk modulus of the drilling mud

$\lambda, \mu \quad$ Eigen values

$\phi \quad$ Bottom slope angle of the channel

$\rho \quad$ Density of the drilling mud

$\theta_{1}, \theta_{2}$ Boundary parameters

$A_{\mathrm{a}} \quad$ Cross sectional area of the annulus

$A_{\mathrm{c}} \quad$ Wetted cross sectional area of the channel

$A_{\mathrm{m}} \quad$ Cross sectional area of the active mud pit

C Measurement matrix of the UKF

$c_{1}, c_{2}$ Design parameters

$F_{\mathrm{a}} \quad$ Friction factor of the annulus

$f_{\mathrm{n}} \quad$ A nonlinear function

$g \quad$ Acceleration of gravity

$h \quad$ Fluid level

$h_{\mathrm{m}} \quad$ Active mud pit level

$I_{1} \quad$ First moment of area

$I_{2} \quad$ Pressure forces in the fluid volume

$J \quad$ Productivity index

$k \quad$ Discrete time index

$k_{\text {choke }}$ Choke coefficient

$l \quad$ Length

$l_{\mathrm{c}} \quad$ Length of the channel

$l_{\mathrm{w}} \quad$ Well depth
A matrix

Number of measurements, UKF

$n_{\mathrm{S}} \quad$ Number of states, UKF

p Pressure

$P_{1}, P_{2}$ Output injection gains

Atmospheric pressure

$p_{\mathrm{r}} \quad$ Reservoir pressure

$Q \quad$ Covariance matrix for process noise

$q \quad$ Volumetric flow rate

$q_{\text {bit }} \quad$ Volumetric flow through the drill bit

$q_{\text {loss }} \quad$ Fluid losses at solid removal equipment

$q_{\text {pump }}$ Mud pump flow rate

$R \quad$ Covariance matrix for measurement noise

$t \quad$ Time

$T_{\mathrm{f}} \quad$ Non-Newtonian friction term

U Control input

$u \quad$ Transformed state, pressure

$v \quad$ Transformed state, flow rate

$v_{k} \quad$ Measurement noise

$w_{k} \quad$ Process noise

$X \quad$ States of the UKF

$x \quad$ Position along the channel length

y Measurement vector

$z \quad$ Position along well depth

$z_{\mathrm{n}} \quad$ Normalized position along well depth

\section{References}

Ole Morten Aamo. Disturbance Rejection in $2 \times 2$ Linear Hyperbolic Systems. IEEE Transactions on Automatic Control, 58(5):1095-1106, 2013. doi:10.1109/TAC.2012.2228035.

Christian Berg. Modeling for Automatic Control and Estimation of Influx and Loss during Drilling Operations. PhD, University of South-Eastern Norway, 2020.

Christian Berg, Anjana Malagalage, Cornelius E Agu, GlennOle Kaasa, Knut Vaagsaether, and Bernt Lie. Modelbased Drilling Fluid FLow Rate Estimation Using Venturi Flume. IFAC-PapersOnLine, 48(6):171-176, 2015. doi:10.1016/j.ifacol.2015.08.027. 
M. Hanif Chaudhry. Open-Channel Flow. Springer, New York, 2nd edition, 2008. ISBN 9780387301747.

Khim Chhantyal, Hakon Viumdal, Saba Mylvaganam, and Geir Elseth. Estimating viscosity of non-Newtonian fluids using support vector regression method: Rheological parameters of drilling fluids using data fusion. SAS 2016 - Sensors Applications Symposium, Proceedings, pages 285-290, 2016. doi:10.1109/SAS.2016.7479860.

Khim Chhantyal, Morten Hansen Jondahl, and Håkon Viumdal. Upstream Ultrasonic Level Based Soft Sensing of Volumetric Flow of non-Newtonian Fluids in Open Venturi Channels. IEEE Sensors Journal, 18(12):5002-5013, 2018. doi:10.1109/JSEN.2018.2831445.

Ven Te Chow. Open-Channel Hydraulics. McGraw-Hill, New York, 1959. ISBN 0070107769.

L. P. Dake. Fundamentals of Reservoir Engineering. Elsevier, Amsterdam, 17th edition, 1998. ISBN 0-444-41830-X.

Mohamed S. Ghidaoui, Ming Zhao, Duncan A. McInnis, and David H. Axworthy. A review of water hammer theory and practice. Applied Mechanics Reviews, 58(1):49-76, jan 2005. doi:10.1115/1.1828050.

Haavard Holta, Henrik Anfinsen, and Ole Morten Aamo. Adaptive Set-Point Regulation of Linear 2x2 Hyperbolic Systems with Uncertain Affine Boundary Condition using Collocated Sensing and Control. In 2017 Asian Control Conference, (ASCC), pages 2766-2771, Australia, 2017. doi:10.1109/ASCC.2017.8287615.

Asanthi Jinasena. Models and Estimators for Flow of Topside Drilling Fluid. PhD Thesis, University of South-Eastern Norway, 2019.

Asanthi Jinasena and Roshan Sharma. Estimation of Mud Losses during the Removal of Drill Cuttings in Oil Drilling. SPE Journal, 2020. doi:10.2118/201230-PA. (in press).

Asanthi Jinasena, Glenn-Ole Kaasa, and Roshan Sharma. Use of Orthogonal Collocation Method for a Dynamic Model of the Flow in a Prismatic Open Channel : For Estimation Purposes. In Proceedings of the 58th Conference on Simulation and Modelling (SIMS 58), pages 90-96, Reykjavik, Iceland, 2017. Linköping University Electronic Press. doi:10.3384/ecp1713890.

Asanthi Jinasena, Ali Ghaderi, and Roshan Sharma. Modeling and Analysis of Fluid Flow through A Non-Prismatic Open Channel with Application to Drilling. Modeling, Identification and Control, 39(4):261-272, 2018. doi:10.4173/mic.2018.4.3.

Asanthi Jinasena, Glenn-Ole Kaasa, and Roshan Sharma. Improved Real-Time Estimation of Return Flow Rate of Drilling Fluids by Model Adaptation for Friction Parameter. IEEE Sensors Journal, 19(20):9314-9323, 2019. doi:10.1109/jsen.2019.2923854.

Morten Hansen Jondahl. Data Driven Models for Estimation of Drilling Fluid Rheological Properties and Flow Rate. PhD Thesis, University of South-Eastern Norway, 2020.
Morten Hansen Jondahl and Håkon Viumdal. Estimating Rheological Properties of Non-Newtonian Drilling Fluids Using Ultrasonic-Through-Transmission Combined with Machine Learning Methods. IEEE International Ultrasonics Symposium, IUS, October(255348):1-4, 2018. doi:10.1109/ULTSYM.2018.8579796.

Morten Hansen Jondahl and Håkon Viumdal. Developing Ultrasonic Soft Sensors to Measure Rheological Properties of Non-Newtonian Drilling Fluids. Technisches Messen, pages 1-14, 2019. doi:10.1515/teme-2019-0039.

Ivan Pirir, Asanthi Jinasena, and Roshan Sharma. A Dynamic Model for Drain Back to Active Mud Pit Combined with a Well Model during Drilling. Journal of Petroleum Science and Engineering, 167:803-818, 2018. doi:10.1016/j.petrol.2018.04.057.

Prasanna Welahettige. Transient drilling fluid flow in Venturi channels : comparing $3 D$ and $1 D$ models to experimental data. PhD Thesis, University of South-Eastern Norway, 2019.

Prasanna Welahettige, Christian Berg, Joachim Lundberg, Bernt Lie, and Knut Vaagsaether. Computational Fluid Dynamics Study of the Effects of Drill Cuttings on the Open Channel Flow. International journal of Chemical Engineering, 2019: $1-9,2019$. 